\title{
Archives délaissées, archives retrouvées, archives explorées : les fonds calédoniens pour l'étude du patrimoine kanak dispersé
}

Neglected archives, encountered archives, explored archives: the Caledonian archives for researches on the scattered Kanak heritage

\section{Marion Bertin}

\section{OpenEdition}

Journals

Édition électronique

URL : http://journals.openedition.org/cel/5438

DOI : $10.4000 /$ cel. 5438

ISSN : 2262-208X

Éditeur

École du Louvre

Référence électronique

Marion Bertin, «Archives délaissées, archives retrouvées, archives explorées : les fonds calédoniens pour l'étude du patrimoine kanak dispersé », Les Cahiers de l'École du Louvre [En ligne], 14 | 2019, mis en ligne le 02 décembre 2019, consulté le 13 décembre 2019. URL : http://journals.openedition.org/ cel/5438; DOl : 10.4000/cel.5438

Ce document a été généré automatiquement le 13 décembre 2019.

\section{(c) (i) $\odot$}

Les Cahiers de l'École du Louvre sont mis à disposition selon les termes de la licence Creative Commons Attribution - Pas d'Utilisation Commerciale - Pas de Modification 4.0 International. 


\title{
Archives délaissées, archives retrouvées, archives explorées : les fonds calédoniens pour l'étude du patrimoine kanak dispersé
}

\author{
Neglected archives, encountered archives, explored archives: the Caledonian \\ archives for researches on the scattered Kanak heritage
}

Marion Bertin

Vu de l'extérieur, le projet de l'Inventaire du Patrimoine Kanak Dispersé demeure le rêve de la plupart des conservateurs et muséographes : bénéficier des ressources permettant de visiter les nombreux musées d'une zone géographique donnée pour faire des recherches sur les collections de son aire d'intérêt et d'expertise. La réalité de la réalisation des inventaires n'était pas forcément aussi séduisante, comme le savent tous ceux qui ont fait des récolements, mais l'émotion ressentie par les professionnels qui ont pu approcher toutes ces pièces dans un cadre privilégié, était toujours présente. Au-delà de l'identification des pièces, l'IPKD a permis d'établir que $85 \%$ des objets et œuvres Kanak en collections publiques dans le monde sont conservés en dehors de la Nouvelle-Calédonie. Cela donne d'autant plus d'importance aux dépôts consentis par les musées Européens, souvent rendus possibles par les liens formés entre professionnels lors de l'inventaire. Loin d'être terminé, l'IPKD continue d'être enrichi régulièrement, et permet à l'heure actuelle la sélection des futurs emprunts destinés à la nouvelle muséographie du projet d'extension et de rénovation du musée de Nouvelle-Calédonie, qui devrait ouvrir en 2021. « Les objets ambassadeurs » du patrimoine Kanak continueront à traverser les océans pour se ressourcer sur leur terre natale. ${ }^{1}$

Marianne Tissandier, conservatrice-restauratrice et responsable des collections du musée de Nouvelle-Calédonie, Nouméa 
1 L'Inventaire du Patrimoine Kanak Dispersé (IPKD) et les « objets ambassadeurs » de la culture kanak sont deux projets mus par une volonté similaire : mieux connaître et promouvoir le patrimoine ancien kanak conservé hors de Nouvelle-Calédonie. Tous deux trouvent leurs racines dans les années 1970, période marquée par un large mouvement identitaire mélanésien. En 1975 est organisé, à Nouméa, Mélanésia 2000, premier festival des arts mélanésiens, dont le comité directeur est présidé par le leader indépendantiste Jean-Marie Tjibaou (1936-1989). Cette manifestation, entendue comme moyen de redonner fierté et confiance aux populations kanak envers leurs pratiques culturelles après plus de deux siècles de domination française douloureuse ${ }^{2}$, marque une véritable « renaissance culturelle kanak $^{3}$ » et un intérêt renouvelé des Kanak pour leur passé. Un Office Culturel Scientifique et Technique Canaque ${ }^{4}$ (OCSTC), placé sous direction gouvernementale française, est créé en 1982 sous l'impulsion de Jean-Marie Tjibaou et Jacques Iékawé (1946-1992). Art, politique et culture sont alors pensés en relation.

2 L'OCSTC joue ensuite un rôle majeur dans la poursuite de recherches sur le patrimoine kanak, dont découlent les projets de l'IPKD et des « objets ambassadeurs ». Jean-Marie Tjibaou, qui en devient le président, s'intéresse rapidement à faire un état des lieux de la création contemporaine et ancienne. En 1979, il rencontre Roger Boulay, jeune ethnologue récemment arrivé en Nouvelle-Calédonie, à qui il confie la première mission de recenser les sculpteurs actifs autour de l'archipel. Notes sur l'état de la sculpture canaque paraît en 1980. Suit un second projet de grande envergure, à nouveau pensé et voulu par Jean-Marie Tjibaou. «L'idée de restitution est une abstraction. On en a beaucoup discuté autrefois avec Jean-Marie Tjibaou : pour lui c'était une abstraction et une pétition de principe qui se heurtait, il le savait très bien, à autant de blocages que d'enthousiasmes aussi peu sensés les uns que les autres. [...] Donc son idée, [...] c'est d'abord de savoir ce qu'il y a : des inventaires, des inventaires, des inventaires. ${ }^{5}$ ”

3 Un portfolio, Les objets kanak dans les musées européens. Première édition 1980-1982, est publié en 1982 sous la direction de Roger Boulay, de l'ethnologue Jean Guiart et de Jacques Iékawé6. Un second volume, Sculptures kanak, paraît en $1984^{7}$. Le projet va plus loin et vise le retour d'objets choisis sur le territoire de la Nouvelle-Calédonie. "S'ils étaient présentés au public dans de bonnes conditions, il n'y avait aucun problème, c'était ça l'essentiel dans son esprit ; par contre, s'ils n'étaient pas utilisés, il proposait non pas d'en demander la restitution mais le dépôt ou le prêt dans le cadre d'une convention. ${ }^{8}$ " Jean-Marie Tjibaou donne à ces objets les noms de "patrimoine kanak dispersé » et d' « objets-ambassadeurs »'. L'expression «objets-ambassadeurs » sera reprise ensuite par les Anciens, personnalités qui bénéficient du droit de parole et d'un haut statut dans les sociétés kanak ${ }^{10}$. Selon Emmanuel Kasarhérou, elle renvoie à l'idée que « ces objets pouvaient être présents autre part que chez nous et constituaient des formes d'ambassadeurs culturels ${ }^{11} »$.

Depuis la création de ces dispositifs, aucun historique détaillé des missions effectuées dans le cadre de l'IPKD et des « objets-ambassadeurs » n'a été réalisé. Les articles sur le sujet furent le plus souvent rédigés par les acteurs des projets eux-mêmes. Roger Boulay ${ }^{12}$ en premier lieu, ainsi qu'Emmanuel Kasarhérou ${ }^{13}$, qui devint le premier conservateur d'origine kanak au musée de Nouvelle-Calédonie (MNC) en 1985, avant de prendre la direction culturelle puis générale de l'Agence de Développement de la Culture Kanak (ADCK) donnèrent également plusieurs conférences à deux voix ${ }^{14}$. Le blog ${ }^{15}$ créé en 2011 par Marianne Tissandier, conservatrice-restauratrice responsable 
des collections du musée de Nouvelle-Calédonie, qui officia quelques mois pour l'IPKD, permit aussi d'en donner une vision quotidienne. De plus, si quelques chercheurs mentionnèrent ces initiatives dans leurs travaux ${ }^{16}$, c'était sans mention des archives conservées à Nouméa qui n'avaient jusqu'à récemment jamais été consultées.

5 Ainsi, grâce à un financement conjoint de l'École du Louvre et de l'Université de La Rochelle, nous avons pu entreprendre un voyage de deux mois et demi en NouvelleCalédonie pour consulter ces fonds d'archives. Nous avons choisi de nous concentrer sur ceux conservés par le MNC et par le Département des Arts Plastiques et des Expositions (DAPEX) de l'ADCK au centre culturel Jean-Marie Tjibaou (CCT) à Nouméa. Nous présenterons ici les archives consultées en insistant principalement sur leur organisation, leur matérialité et leur nature. En effet, la diversité, tant des supports que des contenus, fait la richesse de ces fonds. Elle permet une vision globale des enjeux des projets que nous restituerons ici. Nous mettrons également en valeur la temporalité des initiatives, telle qu'elle est rendue visible à l'aune des documents conservés en Nouvelle-Calédonie. S'y dessine le temps long de la réflexion en regard de missions officielles plus courtes. Les défis et difficultés y sont parfois perceptibles. Enfin, l'implication forte des acteurs des projets donne à ces archives une tonalité personnalisée spécifique, ce qui constitue un autre élément d'intérêt.

\section{Deux corpus d'archives, une même synergie}

6 Les deux projets sont interconnectés et accomplis par les mêmes acteurs, Roger Boulay le premier, rapidement secondé par Emmanuel Kasarhérou. Ils se complètent mutuellement en proposant une valorisation des collections kanak conservées dans des institutions muséales internationales, par leur documentation et leur présentation au public, ajoutés à une recherche de bénéfice pour la Nouvelle-Calédonie et les sociétés Kanak. L'IPDK et les "objets ambassadeurs " relèvent néanmoins de deux entités distinctes, en témoigne la conservation physique des archives en deux lieux différents : le MNC, situé dans le centre-ville de Nouméa, et le CCT, lui-même installé dans la baie de Tina, au nord-est de la ville.

7 Le MNC abrite les archives de l'IPKD, composées de trois types de documents : les résultats des premières missions menées par Roger Boulay dans les années 1980, renseignées sous format papier; le déroulé des recherches de l'IPKD sous format papier; une base informatique sous le logiciel Micromusée résumant l'ensemble des recherches.

8 Au CCT sont préservées les documents liés au projet des « objets-ambassadeurs », sous format papier et numérique.

Le MNC, ouvert dans les années 1900 , est le premier musée du territoire ${ }^{17}$. Il est largement consacré à la présentation d'objets kanak et mélanésiens, bien que des travaux actuellement menés envisagent une refonte totale des espaces pour y intégrer la voix des autres communautés vivant dans l'archipel ${ }^{18}$. À la demande de Jean-Marie Tjibaou, Roger Boulay, en poste au Musée des Arts d'Afrique et d'Océanie (MAAO) à Paris, débute à partir de 1982 une série de visites des musées en France et en Europe, à l'aide des inventaires précédemment effectués par Marie-Charlotte Laroche ${ }^{19}$ et Jean Guiart. Il y recense et photographie les objets kanak conservés, en privilégiant les exemples rares ou absents des collections du musée nouméen. Quarante établissements, majoritairement européens, et leurs objets sont inventoriés auxquels 
s'ajoutent quelques musées du Pacifique, principalement en Australie et NouvelleZélande. Roger Boulay rapporte les préoccupations de Jean-Marie Tjibaou: «Il avait trois questions qui revenaient tout le temps: "Où sont-ils? Comment sont-ils conservés? Que dit-on sur nous ?" ${ }^{20} »$

Ainsi, le MNC conserve un ensemble de plus de mille fiches bristol de format B5, réparties dans cinq boîtes métalliques de rangement (fig. 1), rendant compte de ces premières recherches. Grâce à elles, s'organise une première mise en réseau des musées conservant des objets kanak et des responsables de ces collections. Elles conduisent en outre à la présentation de l'exposition De Jade et de nacre, d'abord à Nouméa entre mars et mai 1990, puis à Paris au MAAO d'octobre à décembre 1990, sous le commissariat de Roger Boulay et d'Emmanuel Kasarhérou. Avant l'arrivée des objets, ce dernier accompagné de son assistant Yves-Béalo Gony entreprennent de rencontrer les Anciens et les représentants coutumiers à travers l'archipel. "C'était la première fois que ces objets revenaient chez nous. Une grande partie de l'organisation de cette exposition a consisté pour moi à faire le tour de nos différentes aires culturelles. Nous en avons huit, nous avons vingt-huit langues et trois cents tribus. La principale fonction a été d'expliquer que ces objets allaient venir, pourquoi ils allaient repartir et de voir quelle pouvait être la réaction des gens vis-à-vis de ces collections. ${ }^{21}$ " Des cérémonies coutumières pour l'ouverture et la fermeture de l'exposition sont organisées, afin que les populations kanak s'approprient le lieu du musée et ces objets anciens, de retour sur le territoire après leur collecte et leur départ en Europe ${ }^{22}$. Quelques objets, du musée d'Aquitaine de Bordeaux, du musée des Beaux-Arts d'Angoulême et du musée de l'Homme, restent en dépôt au MNC où ils sont toujours.

Fig. 1

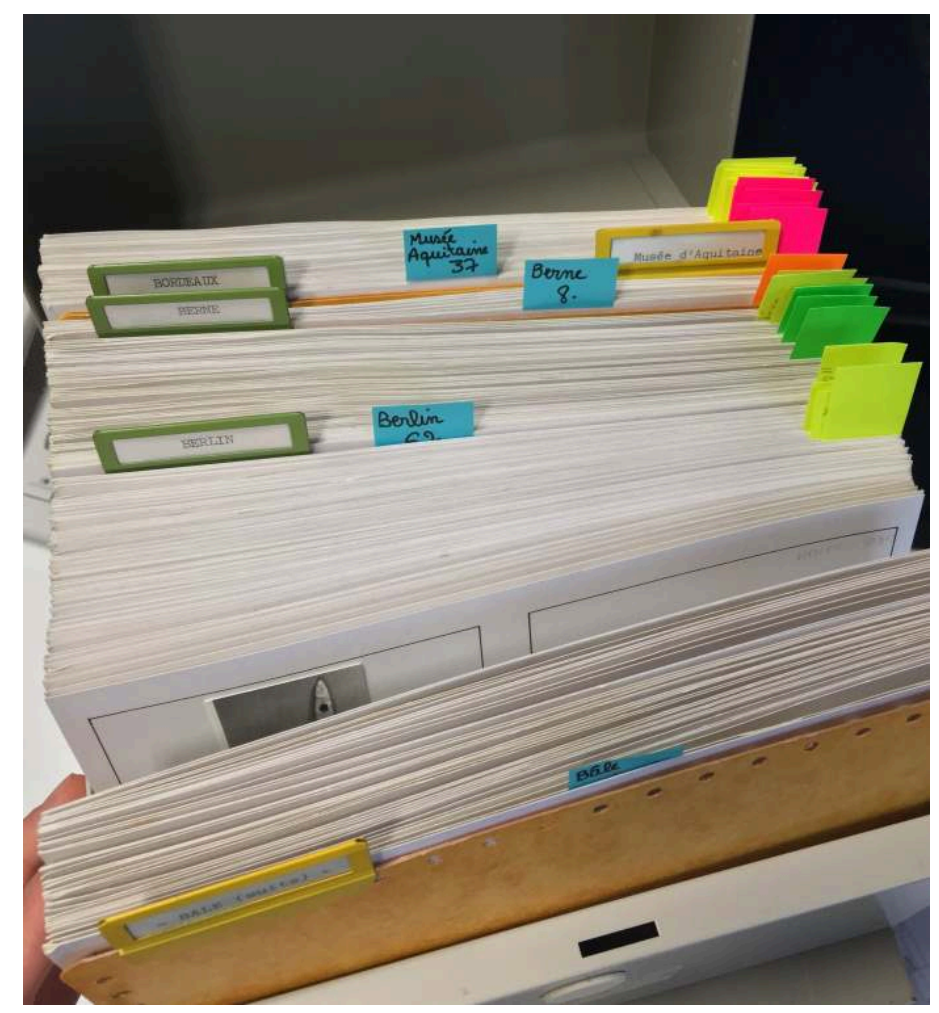

Fiches de Roger Boulay, archives de l'IPKD, musée de Nouvelle-Calédonie. (C) Marion Bertin 
11 D'un point de vue législatif, les accords de Nouméa signés en 1998, qui font suite aux accords de Matignon-Oudinot dix ans auparavant, donnent une nouvelle impulsion aux recherches déjà existantes, en intégrant à l'alinéa 1.3.2 la question des «Objets culturels » : «L'État favorisera le retour en Nouvelle-Calédonie d'objets culturels kanak qui se trouvent dans des musées ou des collections, en France métropolitaine ou dans d'autres pays. Les moyens juridiques dont dispose l'État pour la protection du patrimoine national seront mis en œuvre à cette fin. Des conventions seront passées avec ces institutions pour le retour de ces objets ou leur mise en valeur. ${ }^{23}$ " Cette mesure passe par une meilleure connaissance des objets et en premier lieu leur inventaire. Entre 2011 et 2015 est ainsi créée une mission officielle par le gouvernement de la Nouvelle-Calédonie autour de l'Inventaire du Patrimoine Kanak Dispersé (IPKD). Le siège est fixé à la Maison de Nouvelle-Calédonie à Paris. Quatre membres sont engagés de manière permanente, dont Roger Boulay et Emmanuel Kasarhérou, auxquels s'ajoutent l'historien de l'art Étienne Bertrand et Renée Binosi, secrétaire mise à disposition par la maison de Nouvelle-Calédonie. Plusieurs stagiaires, Marianne Tissandier, Jean-Romaric Néa, Patrice Moasadi, effectuent également des missions de plus courte durée. Une tournée des musées à travers l'Europe est entreprise pendant quatre années. Rangées dans deux cantines métalliques dans les réserves du MNC, cinquante dossiers en carton renferment l'ensemble des archives des musées visités (fig. 2). Les documents relatifs à la préparation de chaque voyage, notamment les correspondances avec les responsables de collections, côtoient divers documents fournis par ces derniers pour appuyer les recherches de l'équipe de l'IPKD, ainsi que les cahiers ayant servi à la prise de notes devant les objets dans les réserves des espaces visités. Quelques ouvrages consacrés aux objets kanak complètent cet ensemble. Plutôt qu'un inventaire exhaustif des objets kanak, l'IPKD vise à la réalisation d'un catalogue raisonné de ces objets anciens. "Par inventaire raisonné, on entend le fait d'inventorier non pas exhaustivement tous les objets de toutes les collections, mais d'inventorier en priorité les objets les plus intéressants. Ces derniers seront choisis en fonction de leur provenance, leur histoire, les circonstances de leur collecte, leur valeur esthétique ou leur état de conservation. En effet, un grand nombre de ces objets présentent un intérêt limité, soit en raison de leur surreprésentation dans les collections - les armes notamment: les sagaies comme les massues -, soit de leur mauvais état de conservation, mais d'autres sont remarquables. ${ }^{24}{ }^{\prime}$ 
Fig. 2

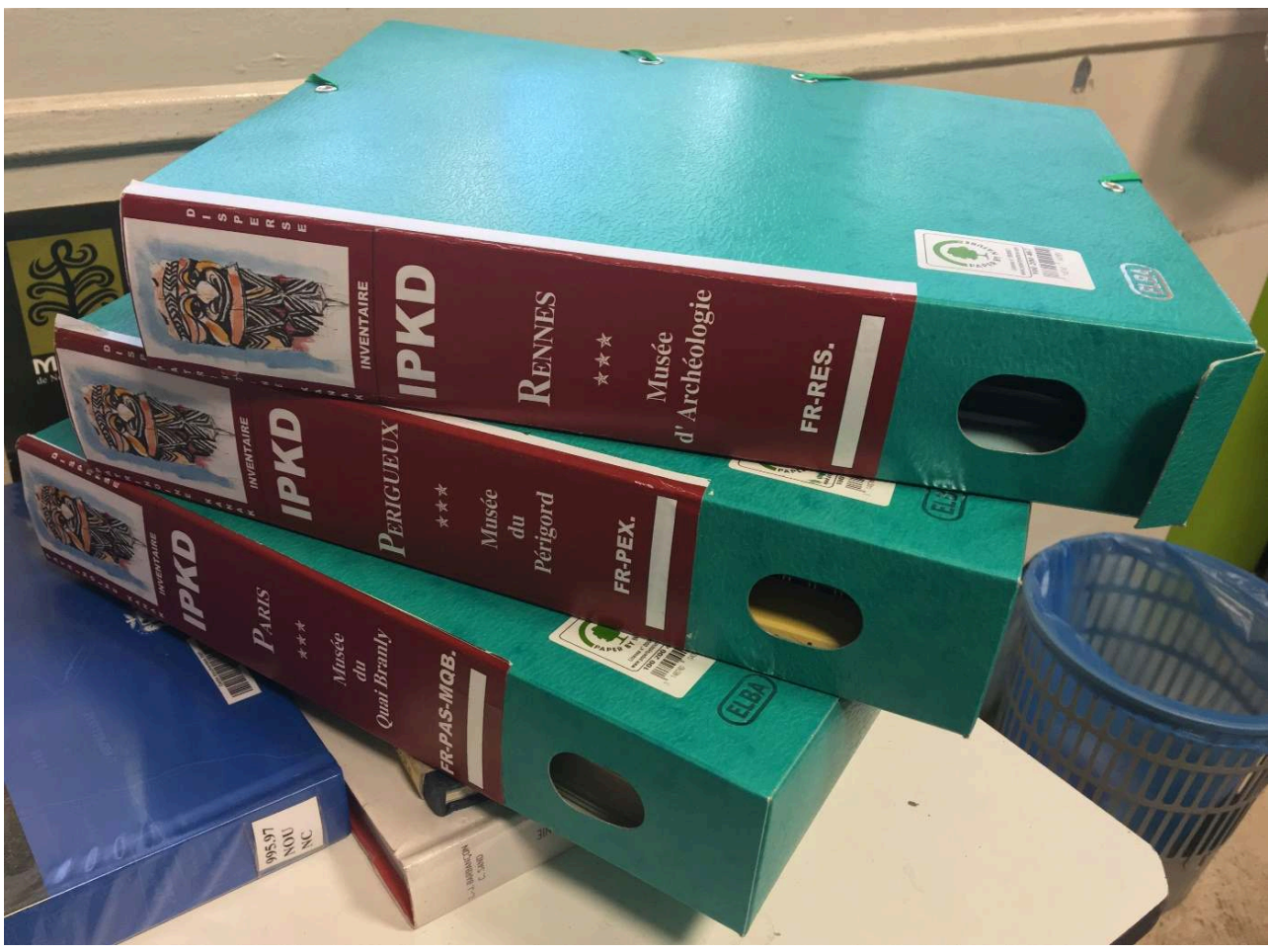

Quelques exemples de boîtes d'archives de la mission IPKD.

(C) Marion Bertin

Enfin, dernier élément et aboutissement de toutes les recherches précédentes, l'IPKD établit une base d'inventaire informatisée sur le logiciel Micromusée. Elle est disponible en intranet au MNC et consultable sur demande. À chaque objet sélectionné correspond une fiche individuelle, incluant des renseignements sur son usage ancien et son histoire, ainsi qu'une photographie. En septembre 2018, à la veille de notre départ de la Nouvelle-Calédonie, la base comptait 5088 notices d'objets, réparties dans 190 musées internationaux et 32 collections privées. Ce décompte n'inclut pas les objets conservés par le MNC, par essence non dispersés.

Si le projet des «objets ambassadeurs" relève quant à lui de l'ADCK, sa chronologie s'entremêle parfois avec celle de l'IPKD, ce qui est aussi le cas des archives. En 1988, au lendemain des Évènements ${ }^{25}$ qui marquent durablement la Nouvelle-Calédonie, les accords de Matignon-Oudinot annoncent la création d'un nouvel établissement entièrement dédié à la présentation de la culture kanak: "Enfin, pour permettre l'expression et l'épanouissement sous toutes ses formes de la personnalité mélanésienne, une action soutenue sera mise en œuvre pour assurer l'accès de tous à l'information et à la culture. À cet effet, il sera créé un établissement public, dénommé "Agence de développement de la culture canaque". ${ }^{26}$ "

L'ADCK est créée l'année suivante, avec l'orthographe "Kanak» choisie par les membres de cette communauté. La construction d'un lieu pérenne, sous la gestion de l'ADCK et dédié à la présentation et à la diffusion des cultures kanak anciennes et contemporaines, s'inscrit dans la politique des «Grands Travaux de la République » menée par François Mitterrand ${ }^{27}$. Il sera l'unique réalisation hors de France métropolitaine. Un terrain sur la presqu'île de Tina, qui accueillit en 1975 le festival Mélanésia 2000, est cédé à titre gratuit par le Conseil municipal de Nouméa en 1991. Le 
centre culturel Jean-Marie Tjibaou ouvre en 1998. L'architecture signée Renzo Piano, sélectionné après un concours international d'architecture, s'apparente à des "souvenirs de $\operatorname{cases}^{28}$ » en s'inspirant de l'architecture kanak traditionnelle. L'ethnologue Alban Bensa joue alors le rôle de conseiller auprès de l'architecte ${ }^{29}$. Dès février 1995, dans un document intitulé «Muséographie des présentations permanentes. Propositions » signé Roger Boulay et Emmanuel Kasarhérou, le projet inclut une salle dédiée au "patrimoine artistique kanak dispersé ${ }^{30}$ ». La case Bwenaado, dont le nom signifie le « rassemblement coutumier » en langue cèmuh $\hat{\imath}^{31}$, première dans le sens de visite du bâtiment est choisie à cet effet. Elle permet ainsi de présenter le patrimoine kanak ancien et d'introduire les autres espaces, tournés vers la production contemporaine. Le retour temporaire d'objets conservés dans des musées internationaux est organisé par un ensemble d'acteurs de l'ADCK, dont Marie-Claude Tjibaou, veuve de Jean-Marie Tjibaou et présidente du conseil d'administration de l'ADCK entre 1989 et 2012, ainsi que les équipe du DAPEX. Le choix final des objets déposés au CCT revient généralement à Roger Boulay, à l'appui des fiches et photographies de recensement du patrimoine kanak dispersé, en concertation avec les équipes de conservation des musées concernés ${ }^{32}$. Des conventions de prestation de service sont signées entre la Réunion des Musées nationaux (RMN ${ }^{33}$ et l'ADCK. Vingttrois objets sont déposés au CCT en 1998, venant de musées allemands, français, suisses et australiens. Ils restent jusqu'en 2001, avant d'être remplacés par des objets alors conservés au musée de l'Homme, puis en 2008 par des objets entrés dans les collections du musée du quai Branly - Jacques Chirac, qui regagnent Paris en 2013. Entre quinze et vingt objets sont à chaque fois concernés. À chaque roulement, après une durée de trois à sept ans, les objets regagnent leurs "musées d'adoption ${ }^{34} »$. Les archives, réparties dans six dossiers et sous format numérique, permettent d'envisager le projet dans ses dimensions scientifiques, logistiques et financières. Le parachèvement du projet des "objets ambassadeurs" et de l'IPKD est la tenue de l'exposition Kanak, l'art est une parole, sous le commissariat de Roger Boulay et d'Emmanuel Kasarhérou, présentée d'abord au musée du quai Branly - Jacques Chirac, puis au CCT de mars à juin 2014. Cent soixante objets sont présentés dans la version nouméenne de l'exposition, dont cent prêtés par des musées hors de Nouvelle-Calédonie. "Ces "objets-ambassadeurs" conservés dans les musées de France et d'Europe, dont la plupart ne sont jamais revenus en Nouvelle-Calédonie, vien[nent] se ressourcer, le temps de cet événement, pour témoigner d'une parole apaisée et offerte à tous ${ }^{35}$. » La fermeture de l'exposition clôt, pour des raisons essentiellement budgétaires et logistiques, le programme des « objets ambassadeurs » malgré son succès local ${ }^{36}$.

\section{Diversité des archives}

La diversité des archives constitue une vraie richesse pour comprendre ces entreprises documentaires. Cette pluralité passe en premier lieu par les multiples supports physiques qui les constituent. Les archives de l'IPKD comportent principalement des fiches bristol, des photographies et négatifs photographiques, une correspondance nombreuse, des cahiers de notes, des articles de journaux, des dépliants et dossiers de presse, divers documents imprimés, des photocopies d'archives anciennes. Hormis la base de données qui est informatisée sur le logiciel Micromusée, les archives ne sont aujourd'hui conservées qu'au format papier. C'est ce format de conservation qui a été privilégié, même pour les dates les plus récentes; des correspondances par courriels et 
des listes d'inventaire extraites de bases de données sont ainsi imprimées et inclues aux archives. On trouve également une bibliographie et quelques ouvrages portant sur des musées et l'histoire des collections, l'histoire de la Nouvelle-Calédonie, des missions scientifiques et des missionnaires y ayant séjourné. À ce jour, la base de données IPKD réalisée sur le logiciel de gestion de collections Micromusée, qui est également celui utilisé au MNC et au CCT, est uniquement accessible depuis le serveur interne du CCT. Sa mise en ligne est évoquée et intègre la poursuite du projet.

Depuis 1998, une partie des archives de l'ADCK-CCT est quant à elle disponible au format numérique. Celles concernant la case Bwenaado sont ainsi partagées, parfois en doublon, entre classeurs et dossiers sous format papier et au format numérique. Les documents peuvent être similaires dans les deux types d'archives ou se compléter mutuellement. Les archives papier sont majoritairement des correspondances entre l'ADCK et différents musées, des listes d'objets et des photographies représentant des objets. Elles intègrent également le livre d'or de l'exposition Kanak, l'art est une parole. Les archives numériques sont d'une grande diversité et combinent des courriels, des documents sous format Word portant sur des projets en cours ou des comptes-rendus de réunions, des factures et devis, des photographies numériques, des articles de presse numérisés, des projets de muséographie pour l'installation des objets dans la case Bwenaado.

17 Cette diversité passe en outre par la nature des contenus qui livrent une vision large des différents aspects des projets menés par la Nouvelle-Calédonie. Les archives de l'IPKD ont un contenu avant tout scientifique, la logistique ayant été assurée dans un premier temps par l'OCSTC pour les premières missions de Roger Boulay, puis par la maison de la Nouvelle-Calédonie à Paris lors de la mission officielle. La correspondance présente dans les archives laisse toutefois entrevoir la logistique sous-jacente à la réalisation du projet.

18 Les archives du DAPEX quant à elles intègrent des problématiques muséographiques, en particulier l'ensemble des propositions de demandes d'« objets ambassadeurs » pour la case Bwenaado. Ces éléments de documentation peuvent prendre la forme de listes d'objets, de modèles de cartels, de plans et vues modélisées des espaces de la case Bwenaado, de correspondances à leur sujet. Ils permettent de suivre l'évolution des objets espérés, demandés et finalement obtenus pour les expositions. Les correspondances ou notes ajoutées sur ces documents sont particulièrement utiles pour cerner les raisons des changements intervenus. Objets déjà requis pour une autre exposition à une date similaire, fragilité de l'objet, dégradations qui nuisent à la lecture de l'objet et sa pleine acception aux exigences de prestige demandées par l'ADCK ${ }^{37}$, restaurations difficiles à envisager, ou contraintes hygrométriques et climatiques font partie des raisons invoquées pour qu'une pièce ne puisse voyager à Nouméa. La présence en grand nombre de ces projets rend parfois difficile à discerner ce qui a été véritablement réalisé.

19 Ces mêmes archives restituent également l'ampleur de la logistique du transport des objets depuis les musées européens vers Nouméa, gérée par la RMN, grâce à des conventions de prestations de services. Opérateur culturel appuyant les musées nationaux ${ }^{38}$, la RMN prête son concours technique et prend en charge les discussions avec les musées prêteurs, ainsi que la coordination et la logistique du transport des objets déposés en Nouvelle-Calédonie, telles que les formalités de douane et d'assurance. Les correspondances, majoritairement par courriels dans ce cas précis, 
indiquent en partie les défis rencontrés autour de la mise en dépôt d'objets à Nouméa. Outre les conventions de dépôts signées entre les établissements muséaux, de nombreuses autres formalités entourent la venue des objets. Au convoiement nécessitant une centralisation des dépôts métropolitains et européens dans la capitale française pour le premier voyage (1998) en plusieurs escales des caisses d'objets ${ }^{39}$, s'ajoutent les modalités d'assurance pour le transport et l'exposition des objets au CCT. Les formalités de douanes sont également lourdes, de nombreux objets kanak comportant des matériaux dont la circulation est aujourd'hui strictement restreinte par la Convention sur le commerce international des espèces de faune et de flore sauvages menacées d'extinction (CITES) de 1973. Les restrictions douanières de la Nouvelle-Calédonie, et notamment l'interdiction d'exporter des objets de plus de cent ans d'âge ${ }^{40}$, requiert l'obtention d'autorisations d'entrées temporaires des objets sur le territoire. L'ADCK et la RMN, dans le cadre de ses missions de prestation, prennent en charge l'ensemble de ces démarches logistiques et administratives. De nombreux documents en rendent ainsi compte dans les archives.

Cette pluralité des types d'archives permet une réelle compréhension de l'organisation administrative et logistique des projets. Elle aide à mieux en saisir les défis, les enjeux et surtout la temporalité.

\section{Temporalité des projets}

21 Les premières recherches et projets mis en place autour du patrimoine kanak dispersé ont débuté à la fin des années 1970. Les résultats de ces quarante années de travail disponibles dans les archives du MNC et du CCT permettent de comprendre leur philosophie, leur réflexion, leur mise en place, leur tenue, leur achèvement et leur épilogue. Le temps long de ces initiatives est ainsi rendu manifeste. En comparaison, la mission gouvernementale IPKD, menée par Roger Boulay et Emmanuel Kasarhérou et bénéficiant d'un budget dédié par le gouvernement de Nouvelle-Calédonie, est plus circonscrite dans le temps et se déroule seulement de 2011 à 2015, ce dont rendent compte des archives stockées séparément. La case Bwenaado abrite quant à elle des objets du patrimoine dispersé pendant seize ans, de 1998 à 2014.

Les archives de l'IPKD rendent compte des différentes strates d'informations, depuis les premières fiches bristol de Roger Boulay. Chacune porte les informations d'identification de l'objet, ainsi qu'une photographie, réalisée généralement dans les réserves d'un musée (Fig. 3 et Fig.4). Certains objets ont été dessinés et peints à l'aquarelle, principalement pour mettre en exergue un détail, en représenter plusieurs angles, ajouter des détails sur les dimensions et les matériaux ou bien présenter des comparaisons de divers objets étudiés. Les photographies ont été numérotées avec une nomenclature tripartite qui intègre l'année de leur prise de vue et offre un suivi, année après année, des musées visités. De nombreux établissements, déjà étudiés par Roger Boulay, ont été à nouveau sollicités par la mission officielle, notamment le Museum für Völkerkunde de Berlin, le Museo Missionario ethnologico Vaticani à Rome, ou le Muséum d'Histoire naturelle de La Rochelle, dont les riches collections méritaient plusieurs séjours de recherches. Les archives de la mission officielle permettent également de comprendre les méthodes de travail de l'équipe chargée de l'IPKD. À chaque musée visité correspond une boîte de rangement, à l'exception de quelques musées dont la faible quantité de documents est rassemblée en une même unité de 
rangement. Ces boîtes, de couleur verte, portent sur la tranche une étiquette marquée du logo officiel de l'IPKD - une applique de case, indiquant le nom du musée, la ville où il est situé et l'abréviation permettant de le désigner dans le classement opéré par l'IPKD (Fig. 2). En effet, au sein de la base de données Micromusée, chaque musée est désigné par une cote correspondant aux premières lettres du pays où il est situé, puis une abréviation de la ville, par exemple «FR-RES» pour le Musée d'archéologie de Rennes. Les différentes boîtes agrègent un ensemble de documents portant sur chaque visite. On y trouve ainsi les éléments ayant servi à préparer la venue des équipes de l'IPDK - correspondances par courriers ou par courriels, documents envoyés en amont par le musée accueillant - et surtout les documents de travail utilisés et réalisés lors de la mission. Reviennent souvent des listes d'inventaire d'objets effectuées par les musées, permettant le repérage et le choix des objets à voir et à photographier, des articles portant sur l'histoire des collections, des dossiers de presse ou documents présentant la programmation du musée. Parfois, des rapports de mission synthétiques rédigées par l'IPKD informent des participants, des contacts dans le musée correspondant, des documents reçus et fournis par ce musée, de personnalités marquantes, des principaux objets kanak, de quelques notes bibliographiques. Enfin, il arrive que des archives rassemblées par Roger Boulay, notamment la correspondance par courriers ou fax, certaines fiches individuelles d'objets réalisées sur papier bristol ou de la documentation ancienne, soient incluses.

Fig. 3

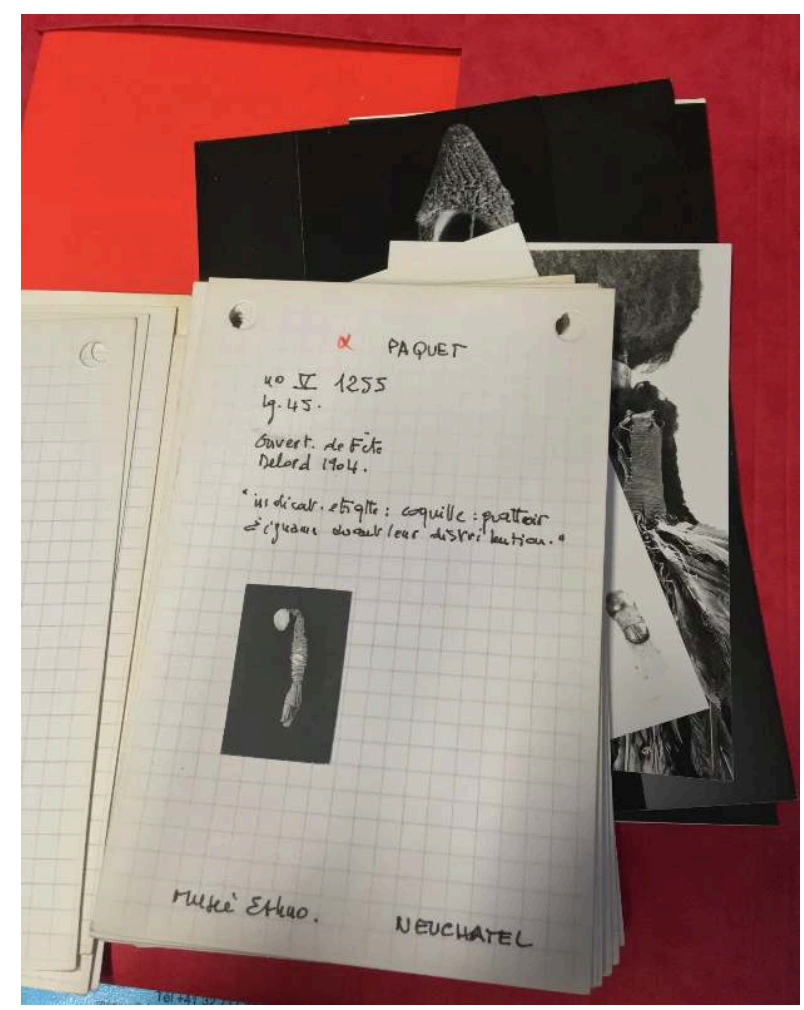

Exemple de fiche de Roger Boulay, archives de I'IPKD.

(c) Marion Bertin 
Fig. 4

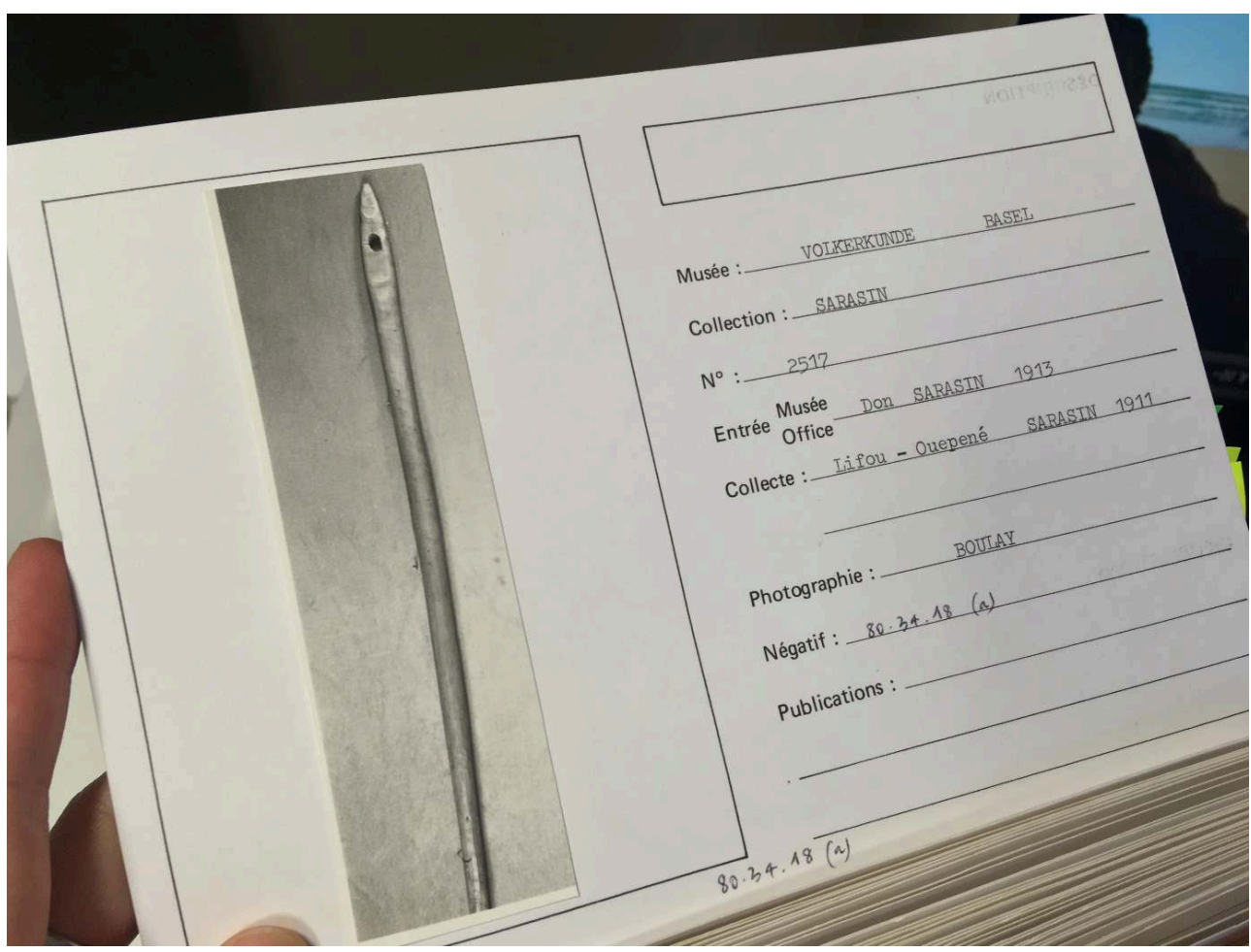

Exemple de fiche de Roger Boulay, archives de I'IPKD.

(c) Marion Bertin

Les documents les plus intéressants pour comprendre la méthode de travail sont les cahiers et feuilles de notes remplis par les équipes de l'IPKD lors de leurs visites de chaque réserve. En effet, ces visites ne pouvaient durer que quelques heures, réparties sur une ou plusieurs journées selon la quantité d'objets à examiner. Des notes sur chaque objet, adjointes de dessins permettant de les reconnaître sur les photographies prises en parallèle, étaient d'abord rédigées sur ces carnets, avant d'être compilées dans la base de données Micromusée. Une micro-fiche individuelle était alors créée pour chaque objet, qui recevait un nouveau numéro d'inventaire spécifique à l'IPKD, construit à partir de l'abréviation des villes et pays et reprenant l'identification de cet objet dans le musée où il est conservé. La base n'est donc qu'une synthèse des recherches réalisées, permettant une indexation et une interrogation des données pour faciliter les recherches, mais nécessitant une consultation des archives pour être pleinement appréhendées et mieux en saisir la genèse. La base de données de l'IPKD intègre toutefois une partie de cette documentation rassemblée, notamment les documents initiaux de Roger Boulay qui ont été en partie numérisés. Il est ainsi possible de mener une archéologie du savoir rassemblé sur les objets kanak.

On retrouve à nouveau l'impact des recherches de Roger Boulay dans le choix des objets demandés en dépôt pour la case Bwenaado du CCT. Des tirages des photographies réalisées par Roger Boulay sont présents dans les archives de l'ADCK ${ }^{41}$ et semblent avoir servi de base pour effectuer un repérage des objets à une époque où les bases de données en ligne des collections muséales étaient encore rares.

Comme nous l'avons évoqué avec le passage aux archives numériques, l'évolution technologique est rendue manifeste par la consultation des fonds. Outre un 
renouvellement des moyens de sauvegarde et de conservation des archives, le développement de l'usage des ordinateurs et d'internet a eu un grand impact sur les possibilités de communication et leur rapidité. L'organisation des premiers dépôts d'œuvres pour le CCT est soumise à certaines difficultés occasionnées par la distance géographique entre Nouméa et l'Europe. Les demandes de dépôt effectuées, en vue de l'ouverture en 1998, sont envoyées par voie postale en août et septembre 1996. Elles sont signées par Marie-Claude Tjibaou. Des lettres de relance sont à nouveau transmises aux musées intéressant l'ADCK en février $1997^{42}$. Les correspondances conservées portent la trace de dialogues parfois difficiles à établir entre les établissements, un délai de plusieurs semaines étant nécessaire pour la transmission du courrier d'un continent à l'autre. Les courriers reçus à l'ADCK semblent indiquer également que plusieurs lettres et fax ont été perdus ou n'ont jamais été reçus. Ces difficultés montrent toute la complexité d'organiser des prêts et mises en dépôt d'objets sur un territoire en marge de la cartographie des grandes expositions. Roger Boulay mentionne également la «lourdeur à la fois institutionnelle et technique des objets $^{43}$ » face aux exigences pour leurs convoiements, même si les conventions de prestation de service signées entre la RMN et l'ADCK facilitent les dialogues avec les musées et la coordination pour la circulation des objets ${ }^{44}$.

\section{Projets institutionnels, rôles individuels}

Les contacts et les relations interpersonnelles établis par Roger Boulay, Emmanuel Kasarhérou et le MNC avec les conservateurs et responsables de collections dans les établissements muséaux d'Europe ont été fondamentaux pour la réussite des projets. Les possibilités de prêts pour l'exposition De Jade et de nacre en 1990, puis les dépôts présentés dans la case Bwenaado au CCT, s'en ressentent. Des visites de musées sont entreprises par Roger Boulay à l'automne 1996, en préparation des premiers dépôts pour la case Bwenaado dont les demandes interviennent en parallèle, afin de faciliter les repérages ${ }^{45}$. Les premiers échanges de courriers concernant ces demandes mentionnent parfois la crainte que ces objets ne restent par la suite à Nouméa et ne soient pas retournés ${ }^{46}$. La salle Bwenaado ne fait pas partie des espaces climatisés du CCT, ce qui a $\mathrm{pu}$ constituer un frein pour certaines institutions et a eu un impact sur le choix des objets mis en dépôt à Nouméa. Des vitrines climatisées et un soin tout particulier ont toutefois été mis en œuvre par les équipes sur place ${ }^{47}$. Emmanuel Kasarhérou a largement insisté sur le "besoin d'une relation de confiance » pour que les prêts d'objets soient acceptés par les musées européens ${ }^{48}$. La bonne entente et l'amitié existant entre les différents protagonistes du projet ont été essentielles, comme le laisse entendre certaines lettres présentes dans les archives. Nous citons ici un extrait d'une lettre adressée par Roger Boulay à Roland Kaehr, alors conservateur des collections océaniennes au musée d'ethnographie de Neuchâtel: «Merci de ton aide ; crois bien que la monnaie du clan d'Emmanuel sera bien gardée et surveillée avec ferveur par l'arrière-petit-fils de son propriétaire. ${ }^{49}$ "

En 2015, un portfolio de planches aquarellées signées de Roger Boulay, représentant des objets importants pour les sociétés kanak recensés dans l'inventaire, est édité et distribué aux musées participants, en remerciements ${ }^{50}$. Les lettres envoyées en retour, conservées dans les archives de l'IPKD, applaudissent le travail réalisés1 ${ }^{2}$, manifestant l'amitié née entre l'ensemble des protagonistes autour de ce projet. 

mêmes. De fait, les archives sont très personnalisées, voire personnelles, par leur association avec leurs producteurs. Ainsi, les premières fiches bristol recensant les objets dans les musées européens et océaniens sont nommées de manière presque officielle comme "les anciennes fiches de Roger Boulay». Au fil des documents consultés, il devient de plus en plus aisé de reconnaître les écritures de Roger Boulay et Emmanuel Kasarhérou, lorsqu'ils ne sont pas signés «RB» et «EK», ou plus généralement « EKS ».

Les projets calédoniens permettent ainsi d'envisager l'importance des individualités pour la bonne poursuite d'initiatives de cette envergure. Si Roger Boulay et Emmanuel Kasarhérou en sont les principaux agents, d'autres protagonistes ont eu une grande importance, à l'exemple d'Irène Bizot, ancienne directrice de la RMN. Si l'interlocutrice principale pour la gestion de ce projet est Marion Mangon, l'influence d'Irène Bizot est perceptible, comme le prouve l'exemple d'une statuette ayant fait partie de la collection de Dominique Vivant Denon (1747-1825), aujourd'hui conservée au musée du quai Branly - Jacques Chirac après avoir fait partie des collections du Musée de Marine du Louvre et du Musée national des arts d'Afrique et d'Océanie (MNAAO) ${ }^{52}$. Demandée en prêt par le musée du Louvre en vue de l'exposition Vivant Denon, L'œil de Napoléon, qui s'est tenue d'octobre 1999 à janvier 2000, elle n'y sera finalement pas présentée car partie en dépôt au CCT dans la case Bwenaado. Des échanges de courriers entre Irène Bizot et Pierre Rosenberg, dont des copies sont conservées dans les archives du CCT, montrent que la première a enjoint au second de retirer sa demande au profit du $\mathrm{CCT}^{53}$. Étienne Bertrand ainsi que les stagiaires ponctuels de l'IPKD - Patrice Moasadi, JeanRomaric Néa, Marianne Tissandier - ont également laissé leurs traces dans les archives de l'IPKD. Ces participants mentionnent un travail en collaboration où les connaissances de chacun étaient valorisées ${ }^{54}$. Pour l'ADCK, Marie-Claude Tjibaou est une personnalité-clé jusqu'en 2012, année où elle quitte la présidence du conseil d'administration, et où l'établissement public est entièrement transféré à la NouvelleCalédonie. Les équipes qui se sont succédées au DAPEX jouent enfin évidemment un grand rôle pour les dépôts et la présentation d'objets dans la case Bwenaado, dont ils ont assuré le travail de régie. Citons tout particulièrement Sandra Maillot Win Nemou pour les premiers dépôts en 1998, Henri Gama, puis Petelo Tuilalo et Anne-Laure Aubail pour les dernières circulations et la tenue de l'exposition Kanak, l'art est une parole en 2014 au CCT. Concernant cette dernière exposition, les équipes du MNC ont apporté une aide majeure au montage. Cela manifeste des relations professionnelles et chaleureuses existant également entre les musées de Nouméa, autour du patrimoine kanak dispersé comme pour d'autres projets.

\section{Conclusion}

La fermeture de l'exposition Kanak, l'art est une parole à Nouméa en juin 2014 marque la fin du programme des «objets ambassadeurs" alors que le CCT doit faire face à des restrictions d'ordre financier, dont est victime la ligne budgétaire auparavant exclusivement allouée à ce programme. Depuis juillet 2018, la case Bwenaado expose un ensemble de sept appliques de case contemporaines conservées par le CCT et le Fonds d'Art Contemporain Kanak et Océanien (FACKO). Elles constituent un nouveau point d'entrée historique, marquant le renouveau de la sculpture kanak à partir des années 
1980, grâce aux efforts de l'OCSTC. La mission de l'IPKD s'achève quant à elle en juillet 2015, après avoir identifié 20000 objets kanak à travers le monde, eu accès à 10000 d'entre eux, mis en fiches 5013 objets et 16000 documents en lien, répertoriés 919 collecteurs et collectionneurs, réalisés 35000 photographies $^{55}$. Depuis, la base de données et sa gestion sont confiées à Marianne Tissandier, qui poursuit le travail de création de notices d'objets.

Ces deux programmes illustrent une «exceptionnelle aventure humaine et muséographique $^{56} »$. Si les archives de Nouméa sont particulièrement riches, elles doivent être également croisées avec celles d'autres acteurs institutionnels. Les archives de la RMN, qui a joué un rôle éminent pour les "objets-ambassadeurs ", en ayant assuré la logistique et leur transport entre l'Europe et Nouméa. Certains musées ont été des partenaires moteurs, depuis les premières recherches menées par Roger Boulay dans les années 1980 jusqu'à l'exposition Kanak, l'art est une parole en 2013-2014. On peut mentionner ici le Museum der Kulturen de Bâle en Suisse, le musée d'Aquitaine de Bordeaux, le musée de l'Homme et le musée du quai Branly - Jacques Chirac à Paris. Enfin, à ces archives conservées par les musées, il faut ajouter l'ensemble des acteurs individuels eux-mêmes, qui sont autant de personnes-ressources importantes pour ces projets. L'ensemble de ces recherches effectuées entre Nouméa et l'Europe sera plus amplement exploité et analysé dans notre thèse de doctorat en cours de rédaction.

\section{NOTES}

1. Marianne Tissandier, conservatrice-restauratrice et responsable des collections du musée de Nouvelle-Calédonie, Nouméa, 19 juillet 2019

2. Caroline Graille, «1975-2015 : retour sur Mélanésia 2000, symbole de la renaissance culturelle kanak », Journal de la Société des Océanistes, 142-143, 2016, pp. 73-98; MarieClaude Tjibaou, Mwà Véé. Revue culturelle kanak, n74/75, 2012, p. 10.

3. C. Graille, « 1975-2015 : retour sur Mélanésia $2000 »$, op. cit.

4. L'orthographe «canaque» a d'abord prévalu, avant d'être réapproprié par cette communauté avec l'orthographe « kanak».

5. Roger Boulay, in Roger Boulay, Marc-Olivier Gonseth, "L'expérience Vanuatu », Tsantsa, vol. 3, 1998, p. 46.

6. Roger Boulay, Les objets kanak dans les musées européens. Première édition 1980-1982, Nouméa, Pacific 2000, 1982.

7. Roger Boulay, Sculptures kanak, Nouméa, Office culturel scientifique et technique canaque, 1984.

8. Roger Boulay, in R. Boulay, M.-O. Gonseth, « L'expérience Vanuatu », op. cit., p. 46.

9. Emmanuel Kasarhérou, lors du séminaire «Le musée comme terrain : stratification des enjeux coloniaux » organisé par l'EHESS et l'ENS, le 12 juin 2018.

10. Roger Boulay, Mwà Véé. Revue culturelle kanak, n74/75, 2012, p. 31. 
11. Emmanuel Kasarhérou lors de la table-ronde «Dialogue des cultures et circulations des œuvres ", Un musée à imaginer. Le musée du quai Branly - Jacques Chirac 10 ans après, musée du quai Branly - Jacques Chirac, 29-30 septembre 2016.

12. Roger Boulay, "De Jade et de nacre », Le Petit Journal des grandes expositions, ${ }^{\circ} 218$, 1990 ; id., "De Jade et de nacre. Patrimoine artistique kanak », Journal de la Société des Océanistes, n90, 1990, pp.57-58; id., Mwà Véé. Revue culturelle kanak, n74/75, 2012, pp. 31-33 ; R. Boulay, M.-O. Gonseth, « L'expérience Vanuatu », op. cit., pp. 45-60 ; Gérard Del Rio, "Patrimoine kanak en région : entretien avec Roger Boulay ", Mwà Véé. Revue culturelle kanak, n54, 2006, pp. 46-49.

13. Gérard Del Rio, Emmanuel Kasarhérou, «Bwenaado: le voyage des "objetsambassadeurs" de la culture kanak entre leurs "musées d'adoption" et la NouvelleCalédonie", Mwà Vée. Revue culturelle kanak, n54, 2006, pp.54-55; Emmanuel Kasarhérou, "Les objets-ambassadeurs de la culture kanak », in Céline Castets-Renard et Guylène Nicolas (dir.), Patrimoine naturel et culturel de la Nouvelle-Calédonie, Paris, L'Harmattan, 2015, pp. 281-285 ; id., « Patrimoine kanak dispersé et culture kanak », in cat. d'exp., Kanak. L'art est une parole, Paris, Musée du quai Branly - Jacques Chirac, octobre 2013 à janvier 2014, Nouméa, Centre culturel Jean-Marie Tjibaou, mars à juin 2014, Paris, Actes Sud \& Musée du quai Branly - Jacques Chirac, 2013, pp. 325-329; id., " The Inventory of Kanak collections in Europe: stolen heritage or common heritage to revive? ", in Sandra Ferracuti (dir.), Elisabetta Frasca (dir.), Vito Lattanzi (dir.), Beyond Modernity. Do ethnography museums need ethnography?, Rome, Espera Libreria Archeologica, 2013, pp. 99-106.

14. Roger Boulay, Emmanuel Kasarhérou, «Un Inventaire du Patrimoine Kanak Dispersé: pour quoi faire?», conférence au centre culturel Jean-Marie Tjibaou, Nouméa, le mai 2012 ; Emmanuel Desbouiges, Dorothée Tromparent, Kanak - Le souffle des ancêtres, Paris, Pao Production et Canal +, 2014 ; Emmanuel Kasarhérou, Roger Boulay, Etienne Bertrand, "Art kanak. Trajectoire d'un inventaire », conférence à la Maison de la Nouvelle-Calédonie, Paris, le 27 octobre 2015 ; Université de la NouvelleCalédonie, Les objets ambassadeurs de la culture kanak, conférence donnée par Roger Boulay, Emmanuel Kasarhérou, Octave Togna. : tv.univ-nc.nc/a400/20120919170358/ [15/07/2019].

15. ipknkd.blogspot.com/ [15/07/2019].

16. Notamment : Jole Cerutti, «Objets et savoirs en mouvement en Nouvelle-Calédonie : histoires et politiques d'un patrimoine partagé », Thesis, n¹7, 2016-2017, pp. 69-92 ; Benoît de l'Estoile, table-ronde lors du colloque Du droit des objets (à disposer d'euxmêmes ?), organisé au Collège de France, le 21 juin 2018.

17. Luc Chevalier, Musée de Nouméa. Guide, Paris, Société des Océanistes, 1975.

18. La rénovation du MNC entre en résonance avec l'idée de « destin commun " prôné par les Accords de Matignon-Oudinot (1988) et de Nouméa (1998). Elle vise ainsi à réconcilier et fortifier les liens entre les communautés habitant sur le territoire.

19. Marie-Charlotte Laroche, "Pour un inventaire des collections océaniennes en France », Journal de la Société des Océanistes, t.1, 1945, pp. 51-57.

20. Roger Boulay : communication personnelle, entretien du 10 septembre 2018.

21. Emmanuel Kasarhérou lors de la table-ronde « Dialogue des cultures et circulations des œuvres ", Un musée à imaginer. Le musée du quai Branly - Jacques Chirac 10 ans après, musée du quai Branly - Jacques Chirac, 29-30 septembre 2016. 
22. R. Boulay, M.-O. Gonseth, « L'expérience Vanuatu », op. cit., p. 48.

23. www.legifrance.gouv.fr/affichTexte.do?cidTexte=JORFTEXT000000555817 [15/07/2019].

24. Personnel $d u$ musée $d u$ Nouvelle-Calédonie: museenouvellecaledonie.nc/ collections/linventaire-du-patrimoine-kanak-disperse-ipkd [15/07/2019].

25. Les « Évènements » désigne une période, de 1984 à 1988, de forte tension entre les communautés du territoire calédonien.

26. www.mncparis.fr/uploads/accords-de-matignon_1.pdf [15/07/2019].

27. Olivier Fandos, Le Centre culturel Tjibaou : histoire du dernier des « Grands Travaux de la République " de François Mitterrand, symbole de l'identité culturelle kanak, Pau, mémoire d'histoire, université de Pau et des pays de l'Andour, 2001.

28. Telles qu'elles furent caractérisées par les représentants coutumiers, Emmanuel Tjibaou : communication personnelle, entretien du 24 juillet 2018. Formule reprise par Renzo Piano: Renzo Piano, "Souvenirs de cases ouverts sur un rêve d'avenir ", in Octave Togna (dir.), Le Centre culturel Tjibaou, Nouméa, Agence de développement de la culture kanak, 1998, pp. 32-43.

29. Alban, Bensa, "L'Ethnologue et l'architecte », in La Fin de l'exotisme, Toulouse, Anacharsis, 2012, pp. 325-339.

30. Nous remercions Roger Boulay de nous avoir communiqué ce document qui ne faisait pas partie des archives liées à Bwenaado consultées à Nouméa. Il n'est pas non plus mentionné dans les archives postérieures à février 1995 que nous avons consultées.

31. La langue cèmuhî est parlée dans la Province Nord de la Grande Terre, dans l'aire coutumière Paici-Camuki, autour des actuelles communes de Koné, Poindimié et Touho.

32. Agence de Développement de la culture Kanak, Archives DAPEX: Dossier non numéroté : Bwenaado + Kannibals et Vahinés.

33. Aujourd'hui Réunion des Musées nationaux - Grand Palais.

34. G. Del Rio, E. Kasarhérou, « Bwenaado : le voyage des "objets ambassadeurs" », op. cit. ,p. 54 .

35. Agence de Développement de la Culture Kanak, archives du DAPEX: Archives numériques non numérotées : Programme Archives 2014 : «Programme 2014 ».

36. Emmanuel Kasarhérou : ww. https://journals.openedition.org/actesbranly/794 [09/10/2019].

37. Pour reprendre les termes de la lettre de Marie-Claude Tjibaou envoyée en guise de demande de prêts d'objets, en août ou septembre 1996 : « Nous souhaitons y montrer de manière temporaire, quelques pièces prestigieuses du patrimoine traditionnel kanak », in Archives de l'ADCK, Archives du Département des Arts Plastiques et des Expositions (DAPEX). Classeur non numéroté : « RMN : Bwenaado Dépôts. »

38. Selon les rôles incombés par le Décret $n^{\circ} 90-1026$ du 14 novembre 1990 relatif à la Réunion des musées nationaux et à l'École du Louvre, avant la transformation de musées nationaux en grands établissements publics dotés de la personnalité juridique.

39. Archives de l'ADCK, Archives du Département des Arts Plastiques et des Expositions (DAPEX). Dossier non numéroté : « Bwenaado + Kannibals et Vahinés. » Chemise : «EKS - Documents de voyage en France, 16.10.2001». 
40. Délibération $n^{\circ} 216$ du 8 novembre 2006 relative aux marchandises soumises à des prohibitions ou à des autorisations administratives d'importation ou d'exportation. La législation actuellement en vigueur a été reprise par l'arrêté n²019-1021/GNC.

41. Archives de l'ADCK, Archives du Département des Arts Plastiques et des Expositions (DAPEX). Dossier non numéroté : « Bwenaado + Kannibals et Vahinés. »

42. Archives de l'ADCK, Archives du Département des Arts Plastiques et des Expositions (DAPEX). Classeur non numéroté : « Dépôts Bwenaado."

43. R. Boulay, M.-O. Gonseth, « L'expérience Vanuatu », op. cit., p. 47.

44. Infra p. 14

45. Lettre de Marie-Claude Tjibaou aux prêteurs potentiels, août-septembre 1996, in Archives de l'ADCK, Archives du Département des Arts Plastiques et des Expositions (DAPEX). Classeur non numéroté : «Bwenaado : Dépôts. »

46. Lettre de Roland Kaehr à Marie-Claude Tjibaou, en date du 18 novembre 1996, in Archives de l'ADCK, Archives du Département des Arts Plastiques et des Expositions (DAPEX). Classeur non numéroté : « Bwenaado : Dépôts. »

47. "Dossier technique de la salle Bwenaado", in Archives du Département des Arts Plastiques et des Expositions (DAPEX). Classeur non numéroté : «RMN : Bwenaado Dépôts ».

48. Emmanuel Kasarhérou lors de la conférence donnée avec Roger Boulay «Un Inventaire du Patrimoine Kanak Dispersé : pour quoi faire? », au centre culturel JeanMarie Tjibaou, Nouméa, le 25 mai 2012.

49. Lettre en date du 12 décembre 1996, conservée in Archives de l'ADCK, Archives du Département des Arts Plastiques et des Expositions (DAPEX). Classeur non numéroté : "Dépôts Bwenaado ".

50. Roger Boulay, Art kanak: savoir-faire traditionnels : planches aquarellées, Paris, Éditions de l'Étrave, 2015.

51. Archives de l'IPKD, musée de Nouvelle-Calédonie, Pochette non numéroté: " Documents divers », chemise « Remerciements ».

52. Il s'agit de la sculpture 72.1952.125 dans la nomenclature actuelle du musée du quai Branly - Jacques Chirac.

53. Échanges de courriers au début de l'année 1998, in Archives du Département des Arts Plastiques et des Expositions (DAPEX). Classeur non numéroté : « RMN : Bwenaado Dépôts ».

54. Jean-Romaric Néa : communication personnelle, entretien du 25 avril 2019.

55. www.mncparis.fr/uploads/2015-10-22-mnc-soire-e-ipkd-livret-v2.pdf [15/07/2019].

56. Selon la formule de Joël Viratelle, directeur de la Maison de Nouvelle-Calédonie : www.mncparis.fr/uploads/2015-10-22-mnc-soire-e-ipkd-livret-v2.pdf [15/07/2019]. 


\section{RÉSUMÉS}

L'Inventaire du Patrimoine Kanak Dispersé (IPKD) et les «objets-ambassadeurs » de la culture kanak sont deux projets mus par une volonté similaire: mieux connaître et promouvoir le patrimoine ancien kanak conservé hors de Nouvelle-Calédonie. Si ces deux initiatives ont été régulièrement citées et commentées par leurs acteurs et différents auteurs, les archives qui les concernent sont toutefois restées relativement méconnues et inexploitées. Conservées au musée de Nouvelle-Calédonie et au centre culturel Tjibaou à Nouméa, elles n'avaient jamais été consultées avant 2018. Un voyage de deux mois et demi en Nouvelle-Calédonie, entre juin et septembre 2018, nous a permis leur recensement et leur consultation. La diversité de ces documents, tant du point de vue de leur matérialité que de leur contenu, rend ces deux fonds particulièrement riches et passionnants. On y lit la philosophie ayant présidé à ces deux projets, leur temporalité, leur organisation administrative. Cet article aura pour but de présenter les deux corpus en insistant davantage sur les documents eux-mêmes plutôt que sur les résultats de leur étude.

The Inventory of the Kanak scattered heritage (Inventaire du Patrimoine Kanak Dispersé, IPKD) and the "Objects as ambassadors" for Kanak culture are two projects with the same goal: to promote and improve knowledge on the ancient Kanak heritage located outside New Caledonia. These two projects were often quoted and commented on by the actors themselves and scholars, but the source material itself has stayed relatively unknown and un-consulted. Preserved by the museum of New Caledonia and by the cultural center Tjibaou in Noumea, these archives had never been considered until 2018. A research trip to New Caledonia for two and a half months gave us the opportunity to create an inventory and consult these archives. The diversity of the documents in the collection makes them a particularly interesting and valuable resource for researchers, and they elucidate the philosophy, timeline, and administrative organisation of the projects. This paper will present the two collections of archives and will focus on the documents themselves, rather than the results of their study.

\section{INDEX}

Keywords : New Caledonia, Kanak, inventory, objects as ambassadors, heritage, Bwenaado, loan, archives

Mots-clés : Nouvelle-Calédonie, Kanak, inventaire, objets-ambassadeurs, patrimoine, Bwenaado, dépôt, archives

\section{AUTEUR}

\section{MARION BERTIN}

Diplômée de l'École du Louvre en histoire de l'art, spécialité « Art du xx siècle », puis en muséologie, Marion Bertin a débuté un doctorat en anthropologie en co-encadrement entre l'École du Louvre, sous la direction de Cecilia Hurley-Griener, et l'Université de La Rochelle, sous la direction de Charles Illouz, en 2016. Elle travaille sur les circulations et les valeurs des objets océaniens dans les collections privées et publiques depuis les années 1980. Ses recherches concernent précisément les différentes valeurs - esthétique, économique, sacrée, patrimoniale qui peuvent être attribuées à un objet en fonction d'un contexte, tel que le marché de l'art dit 
tribal, les collections privées, les musées ou encore le patrimoine. Elle est également secrétaire générale pour le Comité international pour la muséologie de l'ICOM (ICOFOM) et trésorière de l'association CASOAR, dédiée à la diffusion des arts et de l'anthropologie de l'Océanie.

After graduating with degrees in Art history and Museology in the École du Louvre, Marion Bertin started a PhD in Anthropology in the École du Louvre, under the supervision of Cecilia Hurley-Griener, and the Université de La Rochelle, under the supervision of Charles Illouz, in 2016. She studies the circulations and valuation of oceanic objects in private and public collections from the 1980's to the present day. Her research focuses on the different types of values - aesthetic, economic, sacred, patrimonial - that might be used to assess an object depending on its context - the so-called tribal art market, private collections, museums, and heritage. She is an active member of the International Committee for Museology (ICOFOM) and is treasurer for the association CASOAR, which is devoted to promoting arts and anthropology of the Pacific islands. 